\title{
Theoretical Analysis of Wave Function under the Influence of Harmonic Oscillator Potential in BEC Experiments
}

\author{
Noori.H.N. Al-Hashimi; Waleed H Abid ${ }^{1}$; Khalid M. Jiad ${ }^{1}$ \\ Department of Physics; College of Education for pure science, University of Basra; Basra; Iraq \\ ${ }^{1}$ Department of Physics; College of science, University of Basra; Basra; Iraq
}

\begin{abstract}
This paper will focus on theoretical study of BEC in one dimension with weakly attractive interactions in particular in a symmetric harmonic oscillator potential. In this case, the ground state may not be symmetric state, which is in contrast to a BEC with repulsive interaction. The behaviors of the wave function under the influences of different external trapping potentials usually used in experiments that lead to produced Bose-Einstein condensation in ultra cold gases is analysis. Several values of harmonic trapping potentials are used. These analyses give us the overall view of the region of confinement that the external trapping potentials have employed and the influence of some terms in Gross-Pitaevskii equation.
\end{abstract}

Keywords: - Laser cooled atom, BEC atom, Trapping, Quantum Oscillator

\section{INTRODUCTION}

The development of quantum mechanics in the early part of the twentieths century presented sophisticatedly symmetric solution; both massive particles and electromagnetic waves share the same fundamental description, in terms of their wave functions. Within this description one can no longer obtain the phase space coordinates of the particle with absolute certainty, either for a massive particle or a photon. Rather, the wave function $\Psi$ (r) gives the probability amplitude that the massive particle or photon exists at a particular point, $\mathbf{r}$, in space [1]. One of the most precise, and certainly valuable, theoretical tools available for the study of quantum-mechanical systems where quantum-statistical effects are important is second- quantized field theory [2]. This theory has formed the basis for many of the most successful treatments of Bose-Einstein condensed systems. It is worth to mention here that the phenomenon of Bose-Einstein condensation was predicted by Albert Einstein in 1925 [3,4], after generalizing Satyendra Nath Bose's derivation of Planck's distribution for photons [5] to the case of non-interacting massive bosons. Bose-Einstein condensation BEC has been a widely studied research topic among physicists and applied mathematicians since its first experimental realization of (BEC) in ultra cold atomic gases were initially verified by a sequence of experiments in 1995 by Anderson et al. (vapor of rubidium) [6] and Davis et al. (vapor of sodium) [7] that those atoms were confined in magnetic traps and cooled down to low temperatures at an order of micro-Kelvin [8]. For detailed discussion see also [6-7]. In these verifications, theoretical exploration of characteristic trapped potential needs a mathematical model describing those potentials which are used experimentally to produce BEC at very low temperatures. Many different shape of Bose-Einstein condensation have been achieved by using different type of trapping potential, for example cigar-shaped BEC which has been considered an interesting subject especially in coherent atom optics [9-11]. External parabolic potential in highly anisotropic axial symmetry has been used to develop BEC see for example [12-18]. In some literatures, many authors investigated the effect of gravitation [19] by adding the gravitational potential as an external interaction. In this paper, we analyze in one dimension the different forms of trapping potential which are typically used in experiments of BEC. This paper focuses on analysis of the wave function by solving the Gross-Pitaevskii equation in one dimension under the influence of harmonic oscillator trapping potential.

\section{THEORY}

A) Mathematical background

Starting from the many-body Hamiltonian description of the cold atoms, and by allowing for the order parameter or wave function it is possible to reduce the problem, for the condensed fraction only. It is governed by a nonlinear Schrödinger equation, the Gross-Pitaevskii equation (GPE) [20-24]

$\left[-\frac{\hbar^{2}}{2 m} \nabla_{x}^{2}+V_{\text {trap }}(x)+\lambda_{1 D}|\psi(x)|^{2}\right] \psi(x)=\mu \psi(x)$

Where $\hbar$ is the reduced Planck constant, $m$ the mass of the boson, $V_{\text {trap }}$ a trapping potential spatially confining the condensate, and $\mu$ the chemical potential of the condensate, physically, the nonlinearity corresponds to the 
mean field exerted on one boson by all the others and is given, for a condensate of $N$ bosons in one dimension, by

$\lambda_{1 D} \equiv g_{1 D} N=\frac{4 \pi \hbar^{2} a N}{m}$

a here is the scattering length, which varies according to the species of bosons. The energy associated with the wave function $\psi(\mathrm{x})$ is obtained according to [20-24]

$E(\psi)=N \int_{\mathbb{R}}\left[\frac{\hbar^{2}}{2 m}|\nabla \psi(x)|^{2} V_{\text {trap }}|\psi(x)|^{2}+\frac{\lambda_{1 D}}{2}|\psi(x)|^{4}\right] d x$

for the Bose-Einstein condensed system. Here the external trapping potential $V_{\text {trap }}$ is taken to be timeindependent. The GPE "which is a self-consistent mean field nonlinear Schrodinger equation (NLSE)" was first developed independently by Gross [25] and Pitaevskii [26] in 1961 to describe the vortex structure in superfluid. The macroscopic wave function/order parameter is normalized to the total number of particles in the system, which is conserved over time, i.e.

$\int \mid \psi\left(x,\left.t\right|^{2} d \boldsymbol{r}=1\right.$

For ideal (non-interacting) gas, all particles occupy the ground state at $T=0 K^{0}$ and $\psi(x, t)$ in the GPE describes the properties of all $N$ particles in the system. For interacting gas, owing to the inter-particle interaction, not all particles condense into the lowest energy state even at zero temperature. This phenomenon is called the quantum depletion. In a weakly interacting dilute atomic vapor, the main concern in this paper, the non-condensate fraction is very small. The mean field theory can be successfully applied and the quantum depletion can be neglected at zero temperature, assuming a pure BEC in the system. If $V_{\text {trap }}$ is continuous and goes to $+\infty$ at infinity, and if $\lambda_{1 \mathrm{D}} \geq 0$, the ground state of $\mathrm{E}[\psi]$ exists and is unique up to a global phase. In addition, the global phase can be chosen such that $\psi_{\mathrm{GS}}$ is real-valued, and positive on real coordinates. In this work, we will consider cases where the confinement $V_{\text {trap }}$ is so tight in some spatial dimension, the x- axis that the condensate can actually be considered a one-dimensional object. This leads to different representations of the nonlinearity $\lambda$ and the expression for the coupling $g_{I D}$ can be found in Refs [21-23] which we refer to a detailed discussion of the validity of the mean field approximation in this case.

\section{B) Different external trapping potentials}

In early BEC experiments, quadratic harmonic oscillator well was used to trap the atoms. Recently more advanced and complicated traps have been applied for studying BECs in laboratories [27-30]. In this section, we will review several typical trapping potentials which are widely used in current experiments.

I. Three-dimensional (3D) harmonic oscillator potential hop [13]:

$V_{\text {hop }}(\boldsymbol{r})=V_{\text {hop }}(x)+V_{\text {hop }}(y)+V_{\text {hop }}(z) \quad V_{\text {hop }}(\boldsymbol{r})=\frac{m}{2} \omega_{r}^{2} r^{2}, \quad r=x, y, z$

Where, $\omega_{x}, \omega_{y}$, and $\omega_{z}$ are the trapping frequencies in $x$-, $y$-, and $z$-direction respectively.

II. 2D harmonic oscillator $+1 \mathrm{D}$ double well potential dwp (Type I) [13]:

$V_{d w p}^{1}(r)=V_{d w p}^{1}(x)+V_{h o p}(y)+V_{h o p}(z) \quad V_{d w p}^{1}(r)=\frac{m}{2} v_{x}^{4}\left(x^{2}-\hat{a}^{2}\right)^{2}$

Where, $\pm \mathrm{a}^{\wedge}$ are the double $\mathrm{w}$ ell centers along the $\mathrm{x}$-axis, $v_{\mathrm{x}}$ is a given constant with physical dimension $1 /[\mathrm{m}$ $\mathrm{s}]^{1 / 2}$.

III. 2D harmonic oscillator $+1 \mathrm{D}$ double well potential (DWP) (Type II) [13]:

$V_{d w p}^{(2)}(r)=V_{d w p}^{2}(x)+V_{h o p}(y)+V_{h o p}(z) \quad V_{d w p}^{(2)}(r)=\frac{m}{2} \omega_{x}^{2}(|x|-\hat{a})^{2}$

IV. 3D harmonic oscillator + optical lattice potential(OPTLP) [13]:

$V_{\text {hop }}(r)=V_{\text {ho }}(x)+V_{\text {opt }}(x)+V_{\text {opt }}(y)+V_{\text {opt }}(z) \quad V_{\text {opt }}(\tau)=S_{\tau} E_{\tau} \sin ^{2}\left(\hat{q}_{\tau} \tau\right)$

where $\hat{q}_{\tau}=2 \pi / \lambda_{\tau}$ is the angular frequency of the laser beam, with wavelength $\lambda_{\tau}$, that creates the stationary 1D periodic lattice, $\mathrm{E}_{\tau}=\left(\hbar^{2} \hat{q}_{\tau}^{2}\right) / 2 \mathrm{~m}$ is the recoil energy, and $\mathrm{S}_{\tau}$ is a dimensionless parameter characterizing the intensity of the laser beam. The optical lattice potential has periodicity $\mathrm{T}_{\tau}=\pi / \hat{q}_{\tau}=\lambda_{\tau} / 2$ along the $\tau$-axis $(\tau=\mathrm{x} ; \mathrm{y}$; z).

V. 3D box potential [19]:

$V_{\text {box }}(x)=\left\{\begin{array}{cc}0 & 0<x, y, z<L \\ \infty & \text { otherwise }\end{array}\right.$

where $\mathrm{L}$ is the length of the box.

\section{C) Dimensionless External Potential:}

The choices for the scaling parameters $t_{0}$ and $x_{0}$, the dimensionless potential $V(r)$ with $\gamma_{y}=t_{0} \omega_{y}$ and $\gamma_{z}=$ $t_{0} \omega_{z}$, the energy unit $E_{0}=\frac{\hbar}{t_{0}}=\frac{\hbar^{2}}{m r_{0}^{2}}$, and the interaction parameter $\beta=4 \pi a_{s} N / r_{0}$ for different external trapping potentials are given below:

I. $\quad t_{0}=\frac{1}{\omega_{r}}, r_{0}=\sqrt{\hbar / m \omega_{r}}, \quad V(r)=\frac{1}{2}\left(x^{2}+\gamma_{y}^{2} y^{2}+\gamma_{z}^{2} z^{2}\right)$,

II. $2 \mathrm{D}$ harmonic oscillator $+1 \mathrm{D}$ double well potential (type I):

$t_{0}=\left(m / \hbar v_{r}^{4}\right)^{1 / 3}, r_{0}=\left(\hbar / m v_{r}^{2}\right)^{1 / 3}, \quad a=\frac{\hat{a}}{r_{0}}, \quad V(r)=\frac{1}{2}\left[\left(x^{2}-a^{2}\right)^{2}+\gamma_{y}^{2} y^{2}+\gamma_{z}^{2} z^{2}\right]$ 
III. $2 \mathrm{D}$ harmonic oscillator $+1 \mathrm{D}$ double well potential (type II):

$$
t_{0}=1 / \omega_{r}, r_{0}=\sqrt{\hbar / m \omega_{r}}, a=\frac{\hat{a}}{r_{0}}, \quad V(r)=\frac{1}{2}\left((|r|-a \mid)^{2}+\gamma_{y}^{2} y^{2}+\gamma_{z}^{2} z^{2}\right)
$$

IV. 3D harmonic oscillator + optical lattice potential:

$t_{0}=1 / \omega_{r}, \quad r_{0}=\sqrt{\hbar / m \omega_{r}}, \quad k_{r}=2 \pi^{2} r_{0}^{2} S_{\tau} / \lambda_{\tau}^{2}, \quad q_{\tau}=2 \pi r_{0} / \lambda_{\tau} \quad \tau=x, y, z$

$V(r)=\frac{1}{2}\left(x^{2}+\gamma_{y}^{2} y^{2}+\gamma_{z}^{2} z^{2}\right)+k_{x} \sin ^{2}\left(q_{x} x\right)+k_{x y} \sin ^{2}\left(q_{y} y\right)+k_{z} \sin ^{2}\left(q_{z} z\right)$.

V. 3D box potential: $r_{0}=\frac{m L^{2}}{\hbar}, r_{0}=L \quad V(x)=\left\{\begin{array}{lr}0 & 0<x, y, z<1 \\ \infty & \text { otherwise }\end{array}\right.$

\section{RESULT AND DISCUSSION:}

It is worth mentioning here that one can assume the atoms are tightly confined in two directions and can be successfully described by one-dimension by Appling harmonic oscillator potential. A Crank- Nicolson Scheme is employed for solving the Gross-Pitaevskii equation. The time interval used in this solution is 0.00020 and the space step is 0.002500 . The most significant factors which affects this numerical solution is the stability since a constant amplification in one time step turns into an exponential amplification over time. In addition to this classical stability requirement, we would also like that the norm of the system is unchanged. In

the present case this corresponds to conservation of the particle number and that the energy are unchanged. These considerations from the physical properties of the system occasionally do not fulfill the norm and energy preservation properties. Careful adjustments between the time interval and space step will reflect the physical properties of this system is satisfied and the result of this numerical solution can be explained satisfactory. The distribution of the harmonic oscillator potentials for different values of frequencies is shown in figure (1). One can conclude from this figure that the shape of distributions of the potential are not affected by the values of the frequencies; it preserves the parabola like distributions but the area of this distributions are increases as the frequencies of the oscillator potential increase. Changing the harmonic oscillator potential in term of values will reflect definitely on the distribution of the normalized wave function along the $\mathrm{x}$-Axis as shown in figure (2). It seem from this figure that as the value of the harmonic oscillator potential decreases, the distribution of the wave function along the $\mathrm{x}$-axis becomes broader mirroring a Gaussian distribution. The nonlinearity terms has an effect on wave function in term of value and distribution, figure (3) shows the initial distribution of wave function and the distribution after 20000 runs for different values of nonlinearity and fixed value of dimensionless frequency ratio. At the centre of the trapping potential when $\mathrm{x}=0$ there are linear relations between the energy and the chemical potential as shown in figure (4), more over this linear relation is also recorded between the normalized wave function and the energy as shown in figure (5). This linear relation are violated between the energy and frequency ratio (Figure 6) from one side and between the wave function and the chemical potential from other side. The relation between the normalized wave function along the z-direction and trapping potential along the y-direction and the axis of propagation (x-axis) is shown in figure (8) with contour levels of the wave function applied over the surface view. As a conclusion one can say that each terms in Gross-Pitaevskii equation play minor and/or major role in analysis the wave function of the Bose-Einstein condensation, and by careful handling these terms will bring the computational values to a satisfactory experimental one.
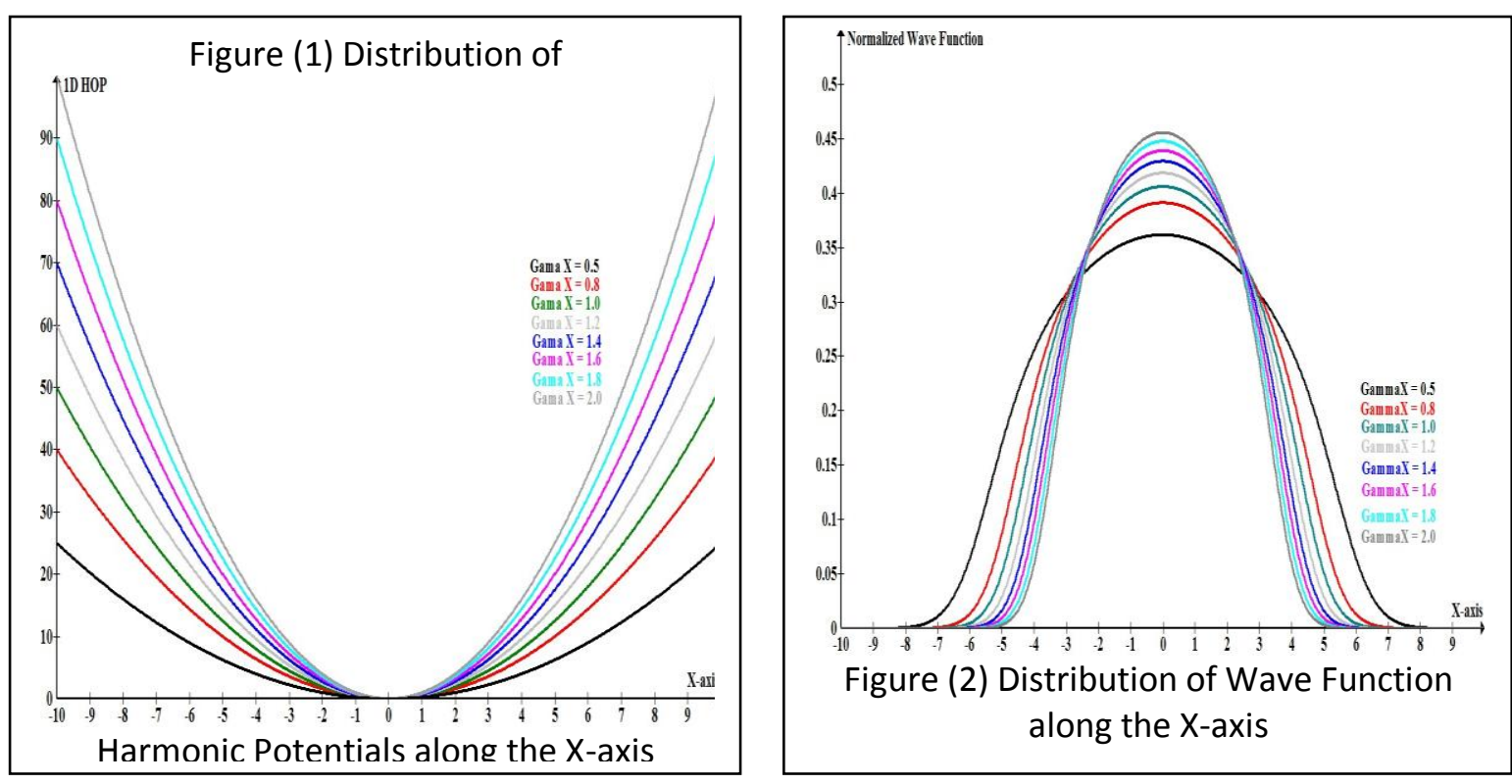

International organization of Scientific Research 


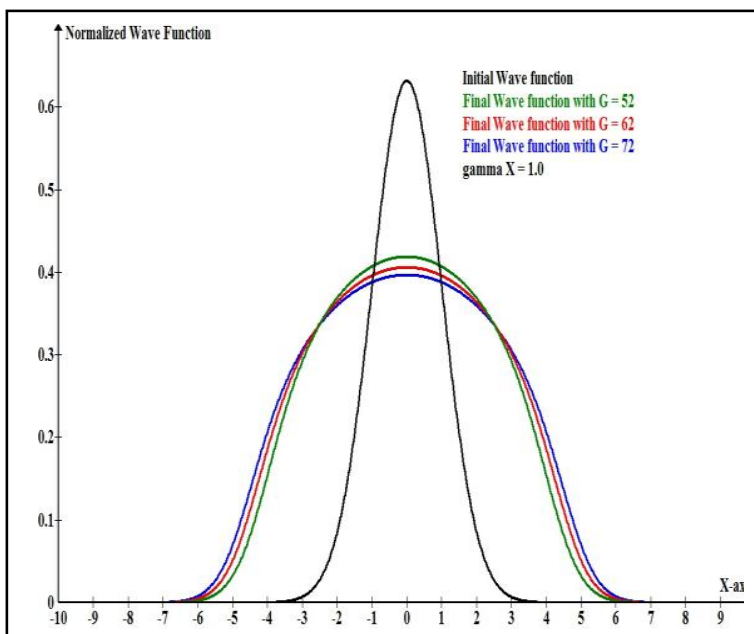

Figure (3) Distribution of Wave Function along the $\mathrm{X}$-axis

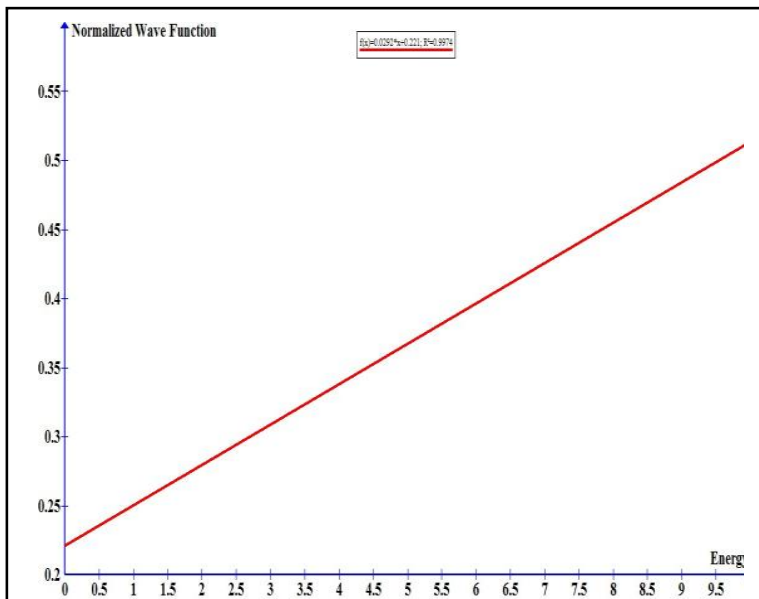

Figure (5) Wave Function as a function of Chemical Potential

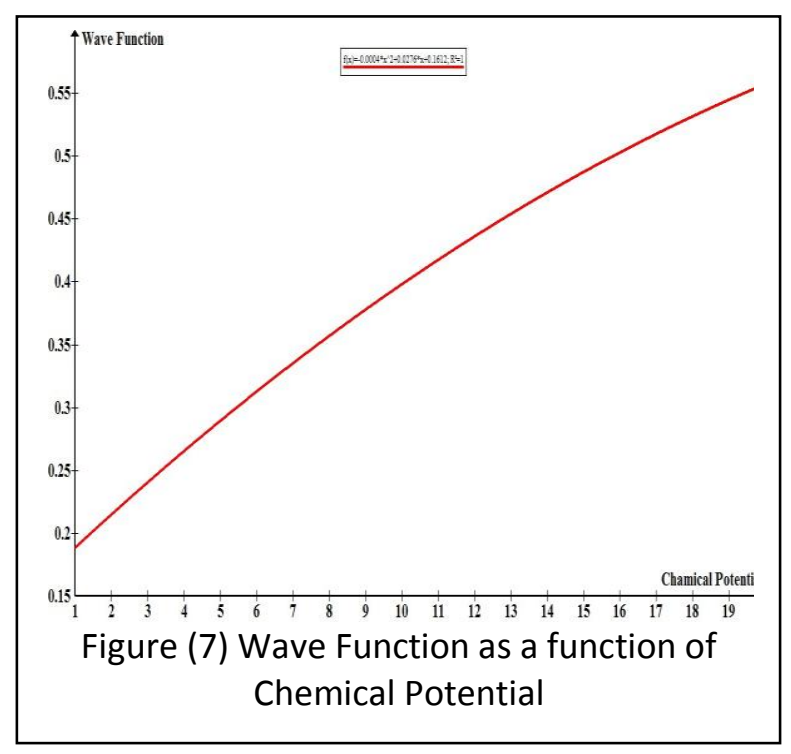

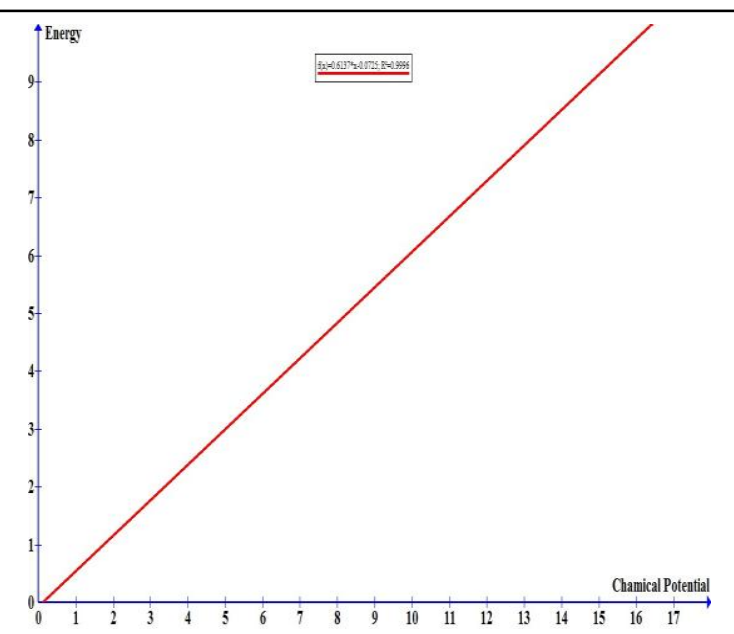

Figure (4) Energy VS Chemical Potentials

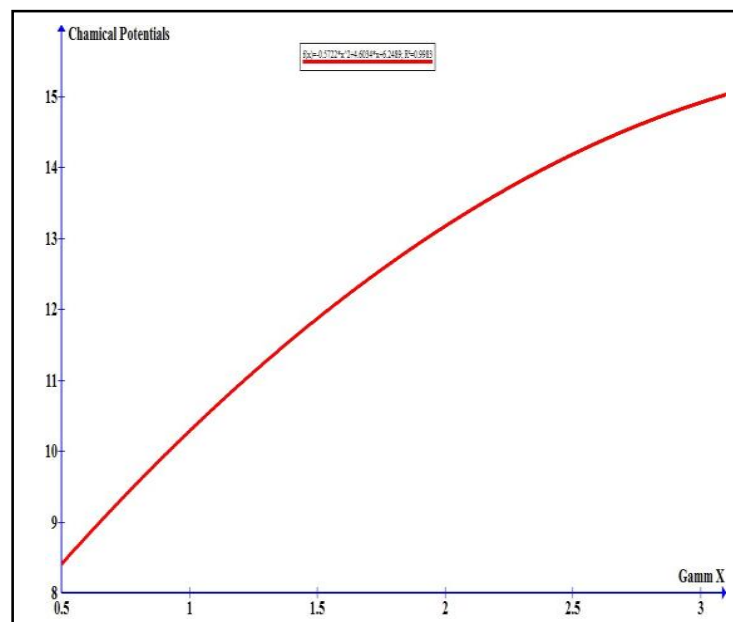

Figure (6) Chamical Potentials as a function of Gamma X

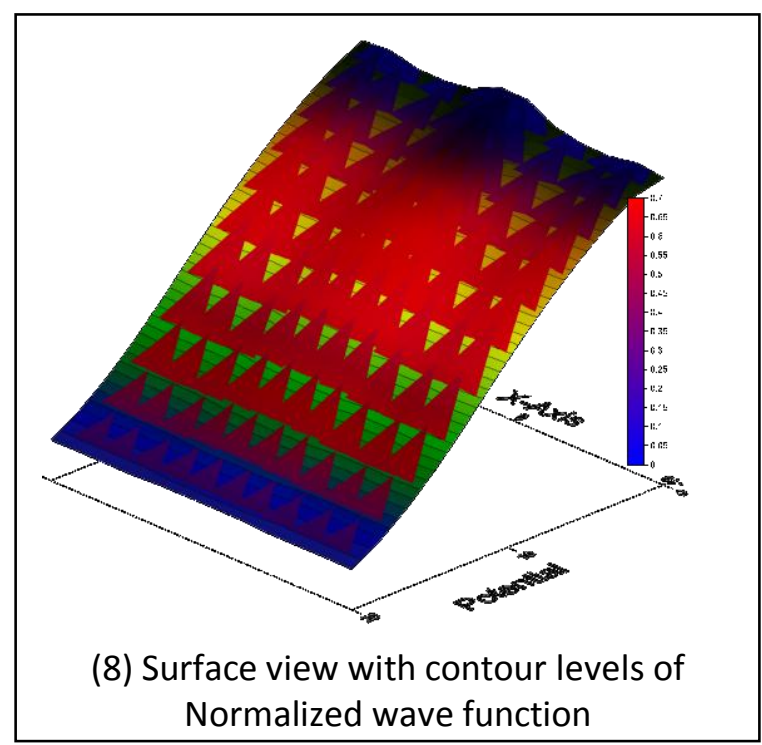




\section{REFRENCES}

[1] Y.J. Wang, D.Z. Anderson, V.M. Bright, E.A. Cornell, Q. Diot, T. Kishimoto, M. Prentiss, R.A. Saravanan, S.R. Segal, S. Wu; " Atom Michelson Interferometer on a Chip Using a Bose-Einstein Condensate" Phys. Rev. Lett. 94 (2005) 090405

[2] A. L. Fetter and J. D. Walecka;" Quantum Theory of Many-Particle Systems" ;(1971) McGraw-Hill.

[3] A. Einstein;"Quantentheorie des einatomigen idealen gases. Sitzungsberichte der Preussischen Akademie der Wissenschaften"; (1924); 22, 261.

[4] A. Einstein;"Quantentheorie des einatomigen idealen gases, zweite abhandlung. Sitzungs- berichte der Preussischen Akademie der Wissenschaften"; (1925); 1, 3.

[5] S. N. Bose.;" Plancks gesetz und lichtquantenhypothese. Zeitschrift fr Physik"; (1924) 3, 178.

[6] M.H. Anderson, J.R. Ensher, M.R. Matthews, C.E. Wieman, E.A. Cornell;" Observation of Bose-Einstein Condensation in a Dilute Atomic Vapor": (1995) Science 269198.

[7] K.B. Davis, M.O. Mewes, M.R. Andrews, N.J. van Druten, D.S. Durfee, D.M. Kurn, W. Ketterle, Phys. Rev. Lett. 75 (1995) 3969.

[8] E.A. Ostrovskaya, Y.S. Kivshar, M. Lisak, B. Hall, F. Cattani, D. Anderson;"Coupled-mode theory for Bose-Einstein condensates"; (2000); Phys. Rev. A 61031601.

[9] W. Ketterle; "When atoms behave as waves: Bose-Einstein condensation and the atom laser"; (2002) Rev. Mod. Phys. Vol.(74) 1131.

[10] Y. Shin, C. Sanner, G.B. Jo, T.A. Pasquini, M. Saba, W. Ketterle, D.E. Pritchard; " Interference of BoseEinstein Condensates on an Atom Chip"; (2005); Phys. Rev. A 72 021604, cond-mat/0506464.

[11] Y. Takasu, K. Maki, K. Komori, T. Takano, K. Honda, M. Kumakura, T.Yabuzaki, and Y. Takahashi; " Spin-Singlet Bose-Einstein Condensation of Two-Electron Atoms"; (2003); Phys. Rev. Lett. 91, 040404

[12] V.M. Perez-Garcia, H. Michinel, H. Herrero,; " Bose-Einstein solitons in highly asymmetric traps"; (1998); Phys. Rev. A 573837.

[13] Noori.H.N. Al-Hashimi and Samer K Ghalib; " Theoretical Analysis of Different External trapping potential used in experimental of BEC ";(2012); IOSR Journal of Engineering (IOSRJEN), Volume 2, Issue 11 PP 01-05

[14] J. Kasprzak, M. Richard, S. Kundermann, A. Baas, P. Jeambrun, J. M. J. Keeling, F. M. Marchetti, M. H. Szymanska, R. Andr'e, J. L. Staehli, V.Savona, P. B. Littlewood, B. Deveaud, and Le Si Dang; "BoseEinstein condensation of exciton polaritons"; (2006); Nature 443, 409.

[15] Y.S. Kivshar, T.J. Alexander, S.K. Turitsyn; "Nonlinear modes of a macroscopic quantum oscillator"; (2001); Phys. Lett. A 278225.

[16] R.A. Duine, H.T.C. Stoof; "Stochastic dynamics of a trapped Bose-Einstein condensate"; (2001); Phys. Rev. A 65013603.

[17] U. Al Khawaja, H.T.C. Stoof, R.G. Hulet, K.E. Strecker, G.B. Partridge;"Bright Soliton Trains of Trapped Bose-Einstein Condensates"; (2002); Phys. Rev. Lett. 89 (2002) 200404.

[18] Jian-Chun Jing and Biao Li; " Exact Analytical Solutions for the (2+1)-Dimensional Generalized VariableCoefficients Gross-Pitaevskii Equation"; (2012); Chinese Journal of Physics VOL. 50, NO. 3 June 2012.

[19] G. Milburn, J. Corney, E. Wright, and D. Walls; "Quantum dynamics of an atomic Bose-Einstein condensate in a double-well potential"; (1997); Phys. Rev. A, 55, 4318.

[20] E.P. Gross,; "Structure of a quantized vortex in boson systems"; (1961) Nuovo Cimento $20454-477$.

[21] L.P. Pitaevskii, Vortex lines in an imperfect Bose gas, Sov. Phys. JETP 13 (1961) 451-454.

[22] F. Dalfovo, S. Giorgini, L.P. Pitaevskii, S. Stringari; "Theory of Bose-Einstein condensation in trapped gases'; (1999); Rev. Mod. Phys. 71 463-512.

[23] S. Stenholm,; "Validity of the Gross-Pitaevskii equation describing bosons in a trap"; (1998); Phys. Rev. A 57 2942-2948.

[24] E.H. Lieb, R. Seiringer, J. Yngvason,; "Bosons in a trap: A rigorous derivation of the Gross-Pitaevskii energy functional"; (2000); Phys. Rev. A 61043602.

[25] M. R. Andrews, M.-O. Mewes, N. J. van Druten, D. S. Durfee, D. M. Kurn, and W. Ketterle, Science 273,84 (1996).

[26] A. Derevianko, W. R. Johnson, M. S. Safronova, and J. F. Babb; " High-Precision Calculations of Dispersion Coefficients, Static Dipole Polarizabilities, and Atom-Wall Interaction Constants for Alkali-Metal Atoms"; (1999); Phys. Rev. Lett. 82, 3589.

[27] J. Bronski, L. Carr, B. Deconinck, J. Kutz, and K. Promislow. "Stability of repulsive Bose- Einstein condensates in a periodic potential"; (2001); Phys. Rev. E, 63, 036612.

[28] L. D. Carr, C. W. Clark, and W. Reinhardt; "Stationary solutions of the one-dimensional nonlinear Schroedinger equation: I. case of repulsive nonlinearity"; (200); Phys. Rev. A, 62, 063610.

[29] Turker Topcu and Andrei Derevianko; Divalent Rydberg atoms in optical lattices: Intensity landscape and magic trapping"; (2014); Phys. Rev. A 89, 023411

[30] L. Pitaevskii and S. Stringari. "Bose-Einstein condensation. Clarendon"; Press, 2003. 